\title{
Hinweise zur Benutzung
}

Die Angaben zur Handschrift $(H)$ sind so gegliedert, dass dem Besitznachweis und der Handschriftenbeschreibung im engeren Sinne (Umfang, Schreiber, Schreibmaterial usw.) Angaben allgemeiner Art folgen, z. B. die Provenienz betreffend. Die Formatangaben beziehen sich auch bei Doppelblättern jeweils auf die Größe des Einzelblatts (Breite $\times$ Höhe in $\mathrm{cm}$ ). Bei Siglen mit Exponenten $\left(h^{1}, h^{2}, E^{1}, E^{2} \ldots\right)$ gelten diese jeweils nur für die Überlieferung des betreffenden Briefes.

Die Formulierung „, Verbleib unbekannt" bedeutet: Die Existenz des Briefes ist sicher, die Handschrift aber nicht nachweisbar. Die Formulierung ,nicht überliefert" ist synonym mit ,verschollen'zu verstehen, das heißt, zum Zeitpunkt des Erscheinens des Bandes ist der Aufbewahrungsort des Briefes den Herausgebern nicht bekannt. Die Formulierung ,vernichtet“ wird nur verwendet, wenn es konkrete Hinweise auf die Vernichtung einer Handschrift gibt. Im Fall der Formulierung ,nicht bekannt" ist es zweifelhaft, ob ein Brief überhaupt existiert hat.

Hinweise auf Faksimiles sind als zusätzliche Information gedacht, ohne dass Vollständigkeit angestrebt wurde. Eine wachsende Zahl der Briefe Goethes, unter anderem die an Charlotte von Stein und an Friedrich Schiller, die im Goethe- und Schiller-Archiv verwahrt werden, stehen als Digitalisate zur Verfügung und sind über das „Repertorium sämtlicher Goethe-Briefe“ im Internet zugänglich (vgl. die Angaben zu GB Rep im Verzeichnis der ,,Siglen und Abkürzungen für Ausgaben und wissenschaftliche Literatur", S. XXIX-LV im vorliegenden Band). Im Rahmen der „PROPYLÄEN. Forschungsplattform zu Goethes Biographica" werden sukzessive weitere Digitalisate veröffentlicht.

Der vorliegende Band enthält Briefe, zu denen außer dem Textzengen, der dem edierten Text zugrunde liegt, Konzepte überliefert sind. Diese werden in einem gesonderten Teil des Textbandes mitgeteilt. Sie tragen die Nummer des dazugehörigen Briefes mit nachgestelltem „K“ (z. B. 95K). Im Unterschied zum edierten Text, dessen Varianten im Hinblick auf die bessere Zitierbarkeit in den Fußnoten mitgeteilt werden, erfolgt die Variantendarstellung der Konzepte in einem integrierten Apparat, doch unter Verwendung derselben Schriftarten, Siglen und Zeichen.

Der Kommentar bietet Überlieferungsvarianten, also Varianten, die nicht auf den Autor selbst zurückgehen, in all den Fällen, in denen ein Brief nicht nach 
einem autorisierten Textzengen, sondern nach einer von mehreren nicht autorisierten Abschriften oder nach einem von mehreren nicht autorisierten Drucken wiedergegeben werden muss. Damit soll der Benutzer in die Lage versetzt werden, die textkritischen Entscheidung der Herausgeber nachzuvollziehen und den Text auch derjenigen Textzengen zu rekonstruieren, die mutmaßlich weniger zuverlässig sind.

Die Erläuterungen folgen dem Grundsatz, dass jeder Brief unter Vermeidung allzu vieler Verweise für sich allein verständlich kommentiert sein soll. Verweise in den Einzelstellenerläuterungen finden in der Regel nur innerhalb eines Bandes statt. Kürzere Erläuterungen werden wiederholt und gelegentliche Redundanzen in Kauf genommen. Verweise in der Form ,vgl. 17,11-12" beziehen sich auf den jeweils vorliegenden Textband (S. 17, Zeile 11-12), Verweise in der Form „vgl. zu 17,11-12 " auf den jeweils vorliegenden Kommentarband, nämlich auf die der Lemmazahl $(17,11-12)$ folgende Erläuterung. Bei Verweisen auf andere Bände tritt jeweils Sigle und Bandzahl davor (vgl. GB 8 II, zu 211,28).

Goethes Werke werden nach der Weimarer Ausgabe zitiert, es sei denn, es gibt eine verbesserte Ausgabe, wie z. B. im Fall von Goethes Autobiographie die von Siegfried Scheibe besorgte Akademie-Ausgabe „Aus meinem Leben. Dichtung und Wahrheit" (AADuW), im Fall der naturwissenschaftlichen Werke die Leopoldina-Ausgabe (LA) und im Fall der Tagebücher und der Begegnungen und Gespräche die am Goethe- und Schiller-Archiv erarbeiteten Ausgaben (GT und BG).

Zitate aus Werken Dritter werden nach den von Goethe benutzten Ausgaben, in der Regel nach deren Erstdruck, nachgewiesen. Sind diese nicht bekannt oder nicht mehr zugänglich, werden andere zeitgenössische oder, wenn vorhanden, historisch-kritische Ausgaben herangezogen.

Bibelstellen sind nach der Ausgabe der Luther-Bibel zitiert, die Goethe selbst besessen hat (Luther-Bibel 1772), weil gelegentlich nicht nur der Nachweis eines Zitats, sondern auch dessen Wortlaut von Bedeutung sein kann.

Fremdsprachige Zitate aus Briefen und Werken werden übersetzt, in der Regel auch fremdsprachige Titel.

Quellen, Werke, Ausgaben und wissenschaftliche Veröffentlichungen, die mehrfach zitiert werden, erhalten eine Sigle oder werden abgekürzt zitiert. Diese Siglen sowie die in Goethes Briefen verwendeten Abkürzungen werden in vorangestellten Verzeichnissen nachgewiesen (vgl. „Siglen und Abkürzungen für Ausgaben und wissenschaftliche Literatur", S. XXIX-LV im vorliegenden Band). 
Geographische Namen und territoriale Bezeichnungen werden nach Maßgabe des damaligen historischen Kontextes wiedergegeben. Gleiches gilt für Amtsbezeichnungen. Wissenschaftliche Begriffe und Zusammenhänge werden auf dem Kenntnisstand der Goethezeit erklärt.

Der Entlastung des Kommentars dienen kommentierte Personen- und Werkregister sowie eine Reihe vorangestellter Verzeichnisse, die sowohl von den Herausgebern als auch von Goethe verwendete Abkürzungen auflösen. 